Check for updates

Cite this: RSC Adv., 2018, 8, 15973

Received 26th March 2018 Accepted 23rd April 2018

DOI: $10.1039 / \mathrm{c} 8 \mathrm{ra02616e}$

rsc.li/rsc-advances

\section{Efficiency of brown seaweed (Sargassum longifolium) polysaccharides encapsulated in nanoemulsion and nanostructured lipid carrier against colon cancer cell lines HCT 116}

\author{
Saghya Infant Shofia, ${ }^{a}$ Kannan Jayakumar, ${ }^{a}$ Amitava Mukherjee (DD ${ }^{\mathrm{b}}$ \\ and Natarajan Chandrasekaran (DD *b
}

Bioactive polysaccharides extracted from brown seaweeds have potent antioxidant, antitumor, antibacterial, antiviral, anti-inflammatory activities and nanomedicine applications. In the present study, we have made an attempt to overcome the instability and bioavailability problem of exopolysaccharides extracted from brown seaweed (Sargassum longifolium) by nanoencapsulation technology to enhance its therapeutic applications. Exopolysaccharides was encapsulated in orange oil nanoemulsion (NE) prepared by ultra-sonication method and nanostructured lipid carrier (NLC) prepared by hot solvent diffusion method. The encapsulation efficiency of nanoemulsion was $67.29 \%$ and of nanostructured lipid carrier was 78.7\%. The prepared nano carriers have particle size $178 \mathrm{~nm}$ (NE), $153 \mathrm{~nm}$ (NLC) and zeta potential $-43.9 \mathrm{mV}(\mathrm{NE}),-60 \mathrm{mV}$ (NLC). In vitro release kinetics of exopolysaccharides from $\mathrm{NE}(80 \%)$ and NLC (95\%) was found to be slow and sustained release which indicates increase in bioavailability. The cytotoxic effect of seaweed polysaccharide, nanocarriers loaded with seaweed polysaccharide was analyzed by MTT method in colon cancer (HCT 116) cell lines with the results revealing that seaweed polysaccharide encapsulated with NLC (80\%) was superior to that encapsulated with orange oil nanoemulsion (70\%). This is the first report demonstrating the potential of brown seaweed exopolysaccharide encapsulated in orange oil nanoemulsion and nanostructured lipid carrier for its biomedical application.

\section{Introduction}

Seaweeds are natural resources with bioactive compounds which are used for food, medical ${ }^{\mathbf{1}}$ and industrial products. ${ }^{2}$ Seaweed is a nutraceutical compound as it is a food with medical and health benefits. Seaweed is an important source of marine polysaccharides that belong to three different families which are classified based on its morphology, cell wall and pigment composition as green (Chlorophyceae), red (Rhodophyceae) and brown (Phaeophyceae) seaweeds. ${ }^{3,4}$ Seaweeds are found in very harsh marine environment with lots of bioactive metabolites with varied functions. For centuries, seaweeds have been used as a food throughout Asia and because of its high nutrient content seaweeds are of high pharmaceutical interest. $^{5,6}$ The seaweed polysaccharides are different from those of terrestrial plant polysaccharides. ${ }^{7}$ They are formed by linking monosaccharides together. The main difference

\footnotetext{
${ }^{a}$ Department of Animal Behaviour and Physiology, School of Biological Sciences, Madurai Kamaraj University, Madurai 625021, India. E-mail: nchandra40@ hotmail.com; nchandrasekaran@vit.ac.in; Tel: +91-416-2202624

${ }^{b}$ Center for Nanobiotechnology, Vellore Institute of Technology, Vellore 632014, India
}

between the seaweed polysaccharides and other polysaccharides is the surface charge. Seaweed polysaccharides are anionic in nature because of the presence of anionic sulfate groups that are absent in polysaccharides of terrestrial origin. ${ }^{8}$ Though seaweeds are composed of various bioactive compounds, $76 \%$ of them are polysaccharides. The structure of those polysaccharides is directly related to the taxonomy and cell structure of seaweed. The major bioactive compounds of seaweeds are polysaccharides and apart from this the other compounds are phenolic, phlorotannins, proteins, peptides, amino acids, terpenes, terpenoids, lipids, and halogenated compounds. Numerous bioactive polysaccharides with interesting functional properties have been discovered from seaweeds. ${ }^{9}$ Polysaccharides from brown seaweeds have been reported to have potent bioactive functions like antiinflammatory activity, anti-oxidant activity and antiproliferative effect on various cancer cells. ${ }^{6}$ Polysaccharides from Sargassum species showed anticancer activity on lung cancer and melanoma, ${ }^{10}$ colon and breast cancer cell lines. ${ }^{\mathbf{1 1 , 1 2}}$ Additionally, polysaccharides derived from marine seaweeds were also used to treat goiter, glandular problems, wounds, burns and rashes. It is also being used in bone replacement 
therapy. Seaweed polysaccharides are used as anticancer agents as it is involved in (i) release of pro-inflammatory cytokines (ii) increase of NK cells activity (iii) apoptosis induction (iv) TLR-4, CD 14 activation. ${ }^{13}$ Seaweed polysaccharides are considered as a source of dietary fiber because they are not completely digested by human intestinal enzymes and thus interfere with the bioavailability of other dietary components. ${ }^{\mathbf{1 4}}$ Seaweeds contain a large amount of carbohydrate as source of polysaccharides. There are several methods to extract polysaccharides. All those are time consuming and they are in need of large amount of organic solvents to extract and precipitate polysaccharides which causes a major problem of environmental pollution. Thus hot water extraction method takes its place by overcoming all these limitations. Hot water extracts of polysaccharides from seaweeds were used in the treatment of cancer by Chinese people. ${ }^{15}$ Since the carbohydrate content in seaweed is considerably high, its greater portion is available as polysaccharide fiber which decreases the bioavailability because it is not taken by the human body. Studies have revealed that high fiber content food can help in reducing the risk of certain cancers, lowers cholesterol and keeps blood sugar balanced. Reports evoke that because of the large size of the macromolecules, a significant portion of it is unable to pass the cell barrier of the gastrointestinal tract. Due to the instability of the nutraceuticals under conditions encountered during processing or in the gut ( $\mathrm{pH}$, enzymes, presence of other nutrients) it is difficult to retain the health benefits of those bioactive molecules. ${ }^{\mathbf{1 6}}$ Therefore, it is necessary to protect these molecules from harsh conditions by encapsulation. Several studies have reported that the bioavailability of the nutraceuticals can be enhanced by increasing the bio accessibility of them during the digestion in small intestine. Nanomedicine is being potently used for rational and targeted delivery for pharmaceutical, therapeutic and diagnostic agents to specific cells and extracellular elements of the body. The mechanism involved in nanoparticles to pass through the gastrointestinal tract barriers includes mainly the particle size. Encapsulation of seaweed polysaccharide increases the efficiency of the bioactive nutraceutical ingredients. Nano carriers with lipids, biosurfactants and water has improved its effectiveness by high entrapment of compound and very low toxic effect because of the components used are generally recognized as safe. ${ }^{17}$ Essential oils are naturally derived aroma compounds synthesized as secondary metabolites from plants. ${ }^{18}$ Orange oil obtained from citrus peel is widely used in the food industries as flavoring agent because of its volatile constituents with unique aroma profiles. Orange oil is a complex organic compound with main components being limonene and linalool which are classified as terpenes. ${ }^{19}$ Nanoemulsions are fine oil-in-water dispersions, having droplet covering the size range from $100-500 \mathrm{~nm} .^{20}$ The essential oils are dispersed in an aqueous medium and stabilized by an emulsifier. Yet another nanoencapsulation technique used is nanostructured lipid carrier (NLC), type of lipid nanocarrier with solid matrix. Advantages of NLC are enhanced drug loading capacity, physical and chemical long term stability, sustained release and prevention of drug expulsion during storage. In our study polysaccharides from Sargassum longifolium was obtained by hot water extraction and ethanol precipitation. Encapsulation of extracted crude polysaccharides was performed using essential oils, solid lipids and biosurfactants for nano carrier formulations. Hence by nanosizing seaweed polysaccharide extracts, it might be efficient enough in bypassing the gastrointestinal tract. Till date no report has been found on nanosizing crude seaweed polysaccharide synthesis by nanoemulsion and NLC technique. Although seaweed polysaccharides have various biological functions, nanosized seaweed polysaccharides have not been investigated yet.

\section{Materials and methods}

\subsection{Materials}

Orange oil, biosurfactants and solid lipid (Span 80, Pluronic L81, lecithin, poloaxmer 188, stearic acid), DMEM, FBS, MTT, DMSO were purchased from Sigma (St. Louis, Mo, USA). Solvents (ethanol, acetone, chloroform, $n$-butanol, acetonitrile, methanol and acetonitrile) were purchased from SRL, INDIA. HCT 116 colon cancer cell lines and 3T3 mouse normal fibroblast cell lines was procured from NCCS (National Centre for Cell Science), Pune, INDIA. All the other chemicals were used of analytical grade.

\subsection{Sample collection and preparation}

Sargassum longifolium, brown seaweed was collected from coastal areas of Gulf of Mannar (Tamil Nadu, India. Latitude $9.1278^{\circ} \mathrm{N}$ and Longitude $79.4662^{\circ} \mathrm{E}$ ) region during low tide in sterile polythene bags with seawater and brought to laboratory. Collected seaweeds were kept in ice and gently washed for few times with sterile distilled water to remove the unwanted debris and dried in air. The air dried seaweeds were grinded into powder and kept in a plastic box covered with aluminum foil for further investigation.

\subsection{Extraction of crude polysaccharides}

Hot water extraction method $^{\mathbf{2 1 , 2 2}}$ was followed with slight modification for extracting polysaccharides from brown seaweed (Sargassum longifolium) extract. Three parameters (Table 1) were studied with different extraction temperature (20, 40, 60, 80 and $\left.100{ }^{\circ} \mathrm{C}\right)$, different $\mathrm{pH}(2,4,7$ and 9) and different extraction time (15, 30, 60 and $120 \mathrm{~min})$ to obtain high yield of crude polysaccharides (Table 2). The pH 2 and 4 were adjusted by addition of $2 \mathrm{~mol} \mathrm{l}^{-1} \mathrm{HCl}$ and $\mathrm{pH} 9$ with $2 \mathrm{~mol} \mathrm{l}^{-1} \mathrm{NaOH}$. Dried Sargassum longifolium seaweed powder $(1: 4 \mathrm{w} / \mathrm{v})$ was boiled in deionized water with the above parameters followed by homogenization using magnetic stirrer to extract the bioactive components from the powder. After extraction, the solution was centrifuged at $7155 \times g$ for $15 \mathrm{~min}$ and supernatant was collected. The extraction process was repeated three times. The supernatants were pooled and concentrated by a rotary evaporator under vacuum at $45^{\circ} \mathrm{C}$. The concentrates were lyophilized with a freeze-dryer. The lyophilized aqueous seaweed extract was prepared in deionized water $\left(1 \mathrm{mg} \mathrm{ml}^{-1}\right)$ and the polysaccharide fraction was precipitated by the addition of three volumes of $100 \%$ ethanol and kept overnight at $-20{ }^{\circ} \mathrm{C}$. The 
Table 1 Polysaccharides yield by following hot water extraction method with different parameters

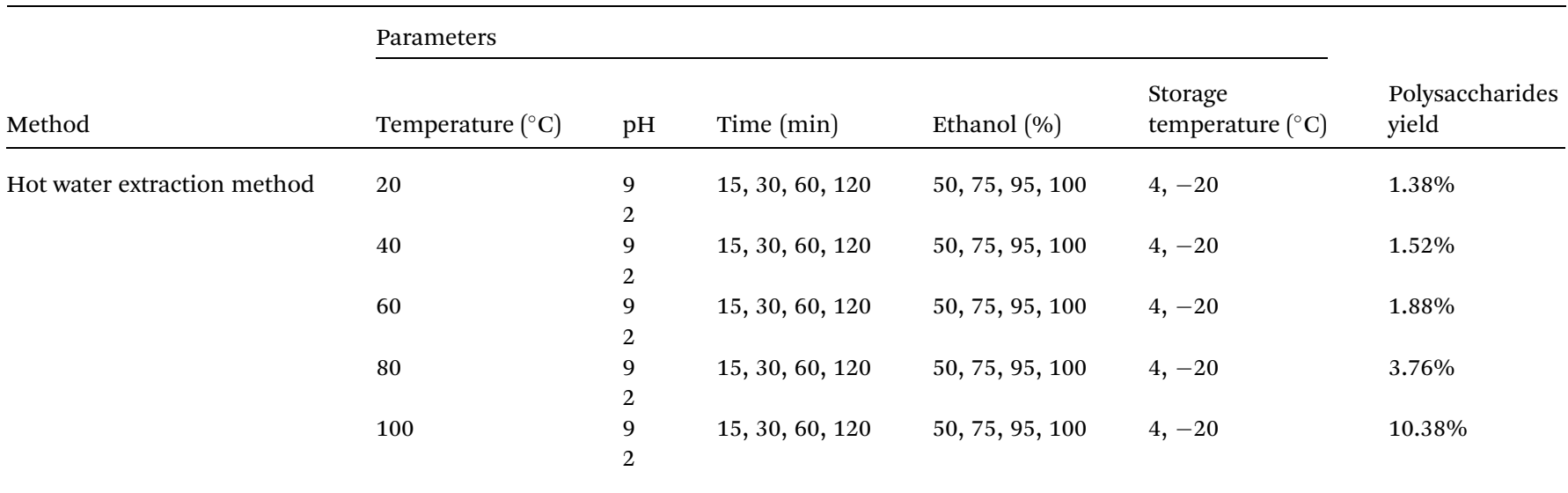

precipitated polysaccharide was collected by centrifugation at $5478 \times g$ for $20 \mathrm{~min}$ at $4{ }^{\circ} \mathrm{C}$ and lyophilized with a freeze dryer to obtain crude seaweed polysaccharide.

\subsection{Deproteinization of crude polysaccharides}

The crude polysaccharides ( $5 \mathrm{mg}$ ) was further dissolved in $10 \mathrm{ml}$ water and partitioned five times with Sevag reagent (chloroform : $n$-butanol, $4: 1$ ) to remove protein. ${ }^{23}$ Two layers were formed from which the aqueous layer was taken carefully and mixed with three volumes of $100 \%$ ethanol and incubated overnight for precipitation at $-20{ }^{\circ} \mathrm{C}$. The precipitated sample was again centrifuged at $5478 \times g$ for $15 \mathrm{~min}$ and lyophilized to obtain dried seaweed polysaccharides.

\subsection{Preparation of nanoemulsion}

Nanoemulsions were prepared with essential oil (orange oil) and biosurfactants (Span 80 and Pluronic L81) by high shear stirring followed by ultra-sonication method at room temperature $^{24}$ (Table 3). The advantage of using both hydrophilic (Pluronic L81) and lipophilic (Span 80) surfactants is to maintain the synergistic effect on emulsion stability. Essential oils and Span $80(10: 1)$ were mixed together to form oil phase, then $1 \%$ Pluronic L81 was dissolved in deionized water to form the water phase by stirring in the beaker. Nanoemulsions were formed by adding the water phase to the oil phase under stirring condition for $30 \mathrm{~min}$ at room temperature. Then the formed emulsion was ultra-sonicated for $30 \mathrm{~min}$ using probe sonicator (30 s ON and $10 \mathrm{~s}$ OFF. 40\% amplitude, 140 WATT) and the reaction mixture was stirred at room temperature for 24 hours. Polysaccharide loaded nanoemulsions were prepared by dissolving $5 \mathrm{mg}$ of crude seaweed polysaccharide to the organic phase followed by other steps as mentioned for blank nanoemulsions. All the prepared nanoemulsions were kept at $25{ }^{\circ} \mathrm{C}$ for further study.

To separate and purify the nanoparticles, 25-30 $\mathrm{ml}$ of acetone was added to break the emulsion followed by bath sonication for $15 \mathrm{~min}$ and centrifuged at $7155 \times g$ for $15 \mathrm{~min}$. Finally the resultant nanoparticles were washed 3-5 times with acetone and water. The samples were then lyophilized to produce polysaccharide nanoparticle in a powdered form.

\subsection{Preparation of nanostructured lipid carrier (NLC)}

Nanostructured lipid carrier was formulated by hot solvent diffusion method ${ }^{25}$ method. Briefly, $20 \mathrm{mg}$ of soy lecithin, $50 \mathrm{mg}$ of stearic acid and $50 \mathrm{mg}$ of orange oil (melted at $72-75^{\circ} \mathrm{C}$ ) were fully dissolved into ethanol : acetone $(40: 60 \mathrm{v} / \mathrm{v})$ mixture at $60{ }^{\circ} \mathrm{C}$ and kept for stirring. The organic solution was then rapidly poured into $50 \mathrm{ml}$ of aqueous solution containing $1 \%$ (w/v) Poloxamer 188 kept under magnetic stirring at $80^{\circ} \mathrm{C}$. The suspension was then cooled to room temperature and the organic solvents were evaporated under reduced pressure using rotary evaporator and the final volumes were adjusted to $20 \mathrm{ml}$. This suspension was filtered through $8 \mu \mathrm{m}$ filter paper and freeze dried to obtain into powder form. The final formulations are shown in Table 3.

\subsection{Nanoparticle characterization}

The $Z$-average, polydispersity index (PDI) and zeta potential was analyzed by dynamic light scattering using Malvern Zetasizer instrument. $^{26-28}$ The mean particle size is a measure of the distribution of nanoparticle population. PDI is a dimensionless measure of the width of the size distribution calculated by the instrument. Zeta potential is a parameter characterizing electrochemical equilibrium on interfaces. Electrostatic repulsion

Table 2 Polysaccharides yield by following hot water extraction method

\begin{tabular}{|c|c|c|c|c|c|c|}
\hline S. no. & Temperature $\left({ }^{\circ} \mathrm{C}\right)$ & $\mathrm{pH}$ & Time (min) & Ethanol (\%) & Storage temperature $\left({ }^{\circ} \mathrm{C}\right)$ & $\begin{array}{l}\text { Polysaccharides } \\
\text { yield }\end{array}$ \\
\hline 1 & 100 & 2 & 180 & 100 & -20 & $10.38 \%$ \\
\hline
\end{tabular}


Table 3 Composition of blank and seaweed polysaccharide (SP) loaded nanoemulsion and NLC formulation

\begin{tabular}{|c|c|c|c|c|c|c|c|c|}
\hline Formulation & $\mathrm{SA}(\mathrm{mg})$ & PO (mg) & OO (mg) & $\begin{array}{l}\text { Span } 80+ \\
\text { Pluronic L61 (\%) }\end{array}$ & Lecithin (mg) & Poloxamer (\%) & Water (ml) & SP (mg) \\
\hline Orange oil & & & 10 & 1 & & & 50 & - \\
\hline NLC P & 50 & & & & 20 & 1 & 50 & - \\
\hline SP NLC P & 50 & & & & 20 & 1 & 50 & 5 \\
\hline
\end{tabular}

between particles depends on the value of zeta potential. Measuring zeta potential is very useful for the assessment of the physical stability of colloidal dispersions. The particle surface develops a charge due to ionization of surface groups or adsorption of ions in the suspension. The charge obtained depend both on surface chemistry of particles and media surrounded by these particles. Measurements were carried out at $25{ }^{\circ} \mathrm{C}$ with a scattering angle of $90^{\circ}$. The samples were diluted about $1: 100$ times in deionized water just before the measurements. All the measurements were performed in triplicate.

\subsection{Scanning electron microscopy (SEM)}

The morphological characteristics of seaweed polysaccharide, nanoemulsions and NLC were examined using scanning electron microscope (EVO-18 plus, Carl Zeiss, Germany). The samples were diluted with deionized water and sonicated for $30 \mathrm{~s}$ in probe sonicator. The samples were prepared by fixing nanoparticles in $\mathrm{KBr}$ pellets were analyzed using an FTIR spectrometer between the range of $500 \mathrm{~cm}^{-1}$ to $4000 \mathrm{~cm}^{-1}$.

\subsection{Entrapment efficiency}

Seaweed polysaccharide entrapment efficiency ${ }^{29}$ in nanoemulsion and NLCs were studied by completely breaking down the NLCs. A volume of $2 \mathrm{ml}$ of seaweed polysaccharide loaded nanoemulsion and NLCs were taken and centrifuged at $12000 \mathrm{rpm}$ for $30 \mathrm{~min}$. The supernatant was then diluted with acetonitrile and methanol mixture $(1: 1) .1 \mathrm{ml}$ of the formulated NLCs were added to $4 \mathrm{ml}$ of and acetonitrile methanol mixture and centrifuged for $20 \mathrm{~min}$ at $8000 \mathrm{rpm}$ and the supernatant was analyzed by UV-VIS spectrophotometer at $490 \mathrm{~nm}$ by Dubois et al. method. The entrapment efficiency was calculated by using eqn (1).

$$
\text { Entrapment efficiency }(\%)=\frac{\text { Total polysaccharide added } \times \text { polysaccharide in the supernatant }}{\text { Total polysaccharide added }} \times 100
$$

one drop of the sample on the glass slide. After air dried, the samples were observed under HR-SEM to evaluate the morphological characteristics.

\subsection{Transmission electron microscopy (TEM)}

The structure of crude polysaccharides loaded orange oil nanoemulsion and NLCs were examined by high resolution transmission electron microscope (HR-TEM, Tecnai T20) using negative staining technique. The nano carrier formulated samples were diluted with deionized water and $10 \mu \mathrm{l}$ of the diluted sample was applied on to the copper grid and air dried for $30 \mathrm{~min}$ at room temperature. For negative staining $10 \mu \mathrm{l}$ of $1 \%$ phosphotungstic acid solution was dropped on the sample grid and allowed to dry at room temperature for $10 \mathrm{~min}$. Photographs were taken at accelerating voltage of $200 \mathrm{kV}$.

\subsection{Fourier transform infrared spectroscopy (FTIR)}

FTIR spectra reveal the characteristic peaks of all the functional groups present in the sample. FTIR spectra of essential oils, seaweed polysaccharide and seaweed polysaccharide

\subsection{In vitro release}

The release of seaweed polysaccharide from the nanoemulsion and NLCs were performed using dialysis membrane bags with 6000-8000 Da pore size. ${ }^{29}$ The dialysis membrane bags were soaked in water overnight ahead of use. The bags were filled with $2 \mathrm{ml}$ of $5 \mathrm{mg} \mathrm{ml}^{-1}$ seaweed polysaccharide nanoparticle loaded NLCs. The bags were immersed in enzyme free simulated intestinal medium (SIM) and simulated gastric fluid medium (SGF) rotated at $50 \mathrm{rpm}$ at $37{ }^{\circ} \mathrm{C}$. A total of $1 \mathrm{ml}$ of sample was taken at sequential time interval followed by replacing the same amount of releasing media soon after withdrawal. The samples were analyzed by UV-VIS spectrophotometer at $490 \mathrm{~nm}$ by Dubois et al. method. For each formulation, the release study was performed in triplicate.

\subsection{Cell culture}

HCT 116 (human colon carcinoma) cell lines and 3T3 mouse normal fibroblast cell lines were cultured and maintained in DMEM supplemented with $10 \%$ (v/v) FBS. The cells were incubated at $37{ }^{\circ} \mathrm{C}$ in a humidified $95 \%$ air or $5 \% \mathrm{CO}_{2}$ environment 

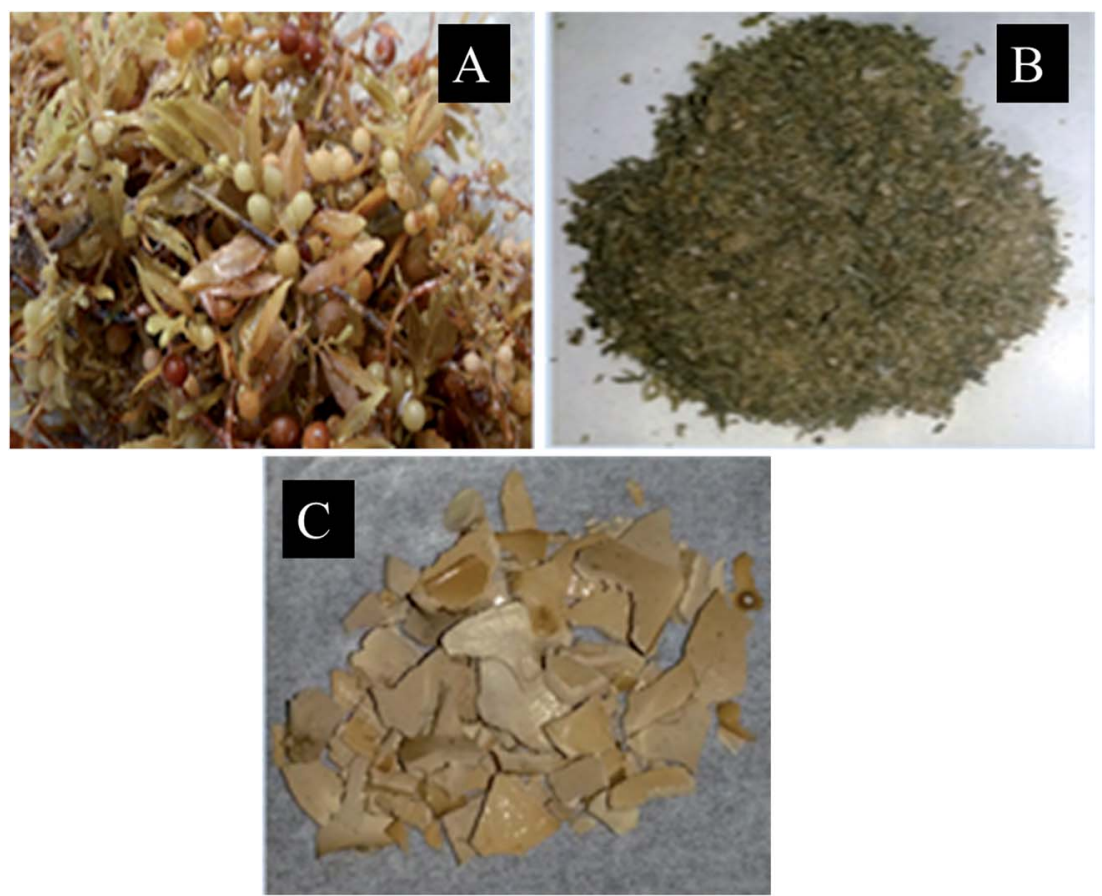

Fig. 1 (A) Sargassum longifolium, (B) ground seaweed powder and (C) as extracted seaweed polysaccharide.

by seeding into 96 well plates at the density of 200000 cells per wells in the media. Different concentration of seaweed polysaccharide and the same loaded into nanoemulsion and NLC were dissolved in deionized water and sonicated for one hour to get rid of aggregation.

The medium with $10 \%$ FBS was removed after $8 \mathrm{~h}$, and replaced by $20 \mu \mathrm{l}$ of samples from low to high concentrations. Controls were cells treated with an equal volume of serum free medium without any samples. Cells were incubated for another $48 \mathrm{~h}$ before the cell viability assay was performed.

\subsection{Mitochondrial activity}

In vitro cytotoxicity ${ }^{30}$ of seaweed polysaccharide and the same loaded into nanoemulsion and NLC was assessed using the MTT (3-(4,5-dimethylthiazol-2-yl)-2,5-diphenyl tetrazoniumbromide) assay to measure the succinate dehydrogenase mitochondrial activity. Fresh media with $100 \mu \mathrm{l}$ of $5 \mathrm{mg} \mathrm{ml}^{-1}$ MTT stain was pipetted into every well and incubated for $4 \mathrm{~h}$. The media was removed and the formation purple color crystals were further dissolved with DMSO. The absorbance was read at $570 \mathrm{~nm}$ in microplate reader (BIOTEK, POWER WAVE XS2). Blank was kept as medium without cells and negative control as medium without samples. All experiments were repeated thrice to ensure reproducibility. Cell viability was expressed as the percent absorbance relative to that obtained for cells not exposed to samples.

\section{Results}

\subsection{Yield of crude polysaccharide}

Sargassum longifolium (Fig. 1A) belonging to the class Phaeophyceae was shade dried and ground into powder (Fig. 1B) to extract polysaccharides (Fig. 1C) using hot water extraction method. The yield of crude polysaccharides obtained from hot water extraction was showed in Table 1 . The extraction process gave different yields of crude polysaccharides depending on the heating temperature, $\mathrm{pH}$, extraction time, ethanol concentration and storage temperature. The highest yield was $10.38 \%$ with heating temperature $100^{\circ} \mathrm{C}, \mathrm{pH} 2$, extraction time $180 \mathrm{~min}$, $100 \%$ absolute ethanol for precipitation and $-20^{\circ} \mathrm{C}$ for storage.

\subsection{Encapsulation of crude polysaccharides in nanocarriers}

Different ratios of orange oil Span 80 and Pluronic L81 were used to find out the suitable nanoemulsion formation. Similarly different ratios of stearic acid, orange oil, lecithin and Poloaxmer 188 were formulated for NLC. The optimized ratios for both the nanocarriers are demonstrated in Table 3. The concentration of crude polysaccharides was kept constant for both formulations as $5 \mathrm{mg} \mathrm{ml}^{-1}$.

\subsection{Physiochemical characteristics of nanocarriers encapsulated with crude polysaccharides}

The $Z$-average, polydispersity index (PI) and zeta potential of the formulated nanocarriers was obtained by dynamic light

Table 4 Particle size, PDI, zeta potential and entrapment efficiency of the formulations

\begin{tabular}{lllll}
\hline Formulation & Size $(\mathrm{nm})$ & PDI & Zeta potential & EE (\%) \\
\hline Orange oil & 111 & 0.564 & $-39.2 \mathrm{mV}$ & \\
$\mathrm{SP}^{a}$ orange oil & 178.7 & 0.009 & $-43.9 \mathrm{mV}$ & 67.29 \\
$\mathrm{NLC}^{a}$ & 123 & 0.29 & $-59 \mathrm{mV}$ & \\
$\mathrm{SP}^{a} \mathrm{NLC}$ & 153 & 0.18 & $-60 \mathrm{mV}$ & 78.7
\end{tabular}

${ }^{a} \mathrm{SP}$ - seaweed polysaccharide. 


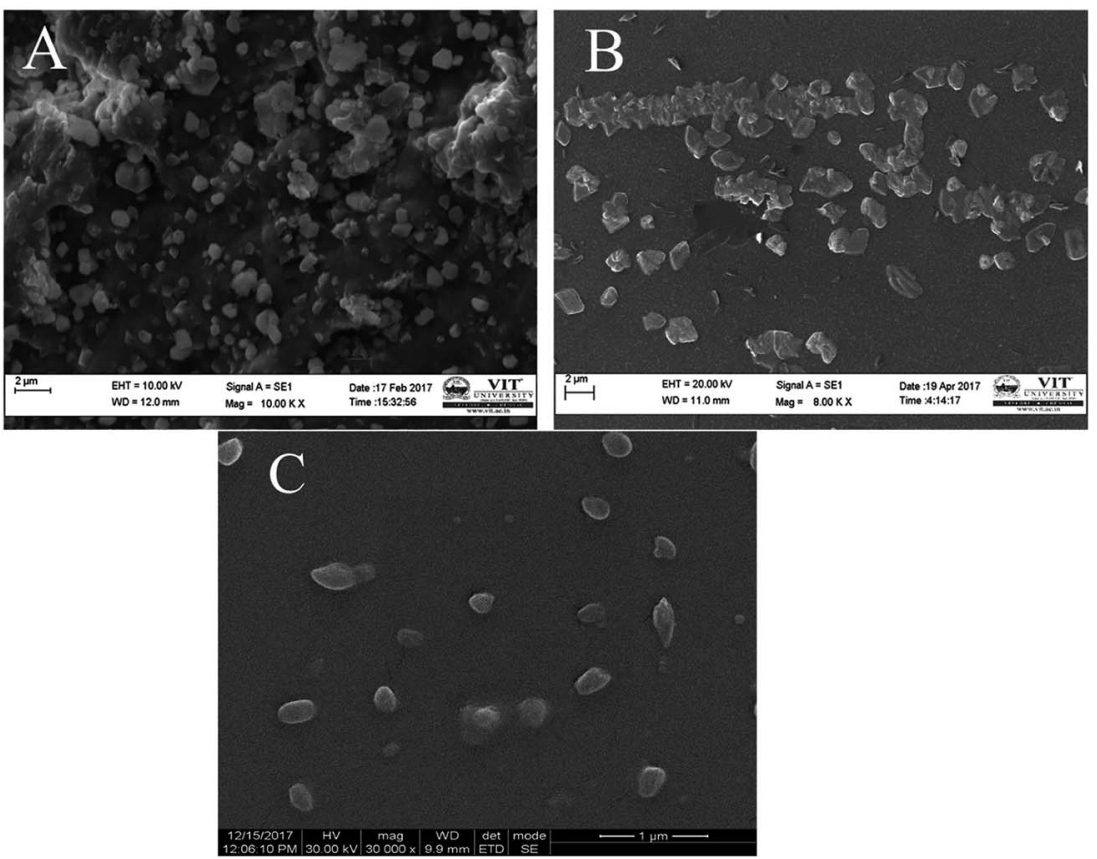

Fig. 2 SEM image of (A) as extracted seaweed polysaccharide (B) orange oil nanoemulsion encapsulated with seaweed polysaccharide (C) NLC encapsulated with seaweed polysaccharide.
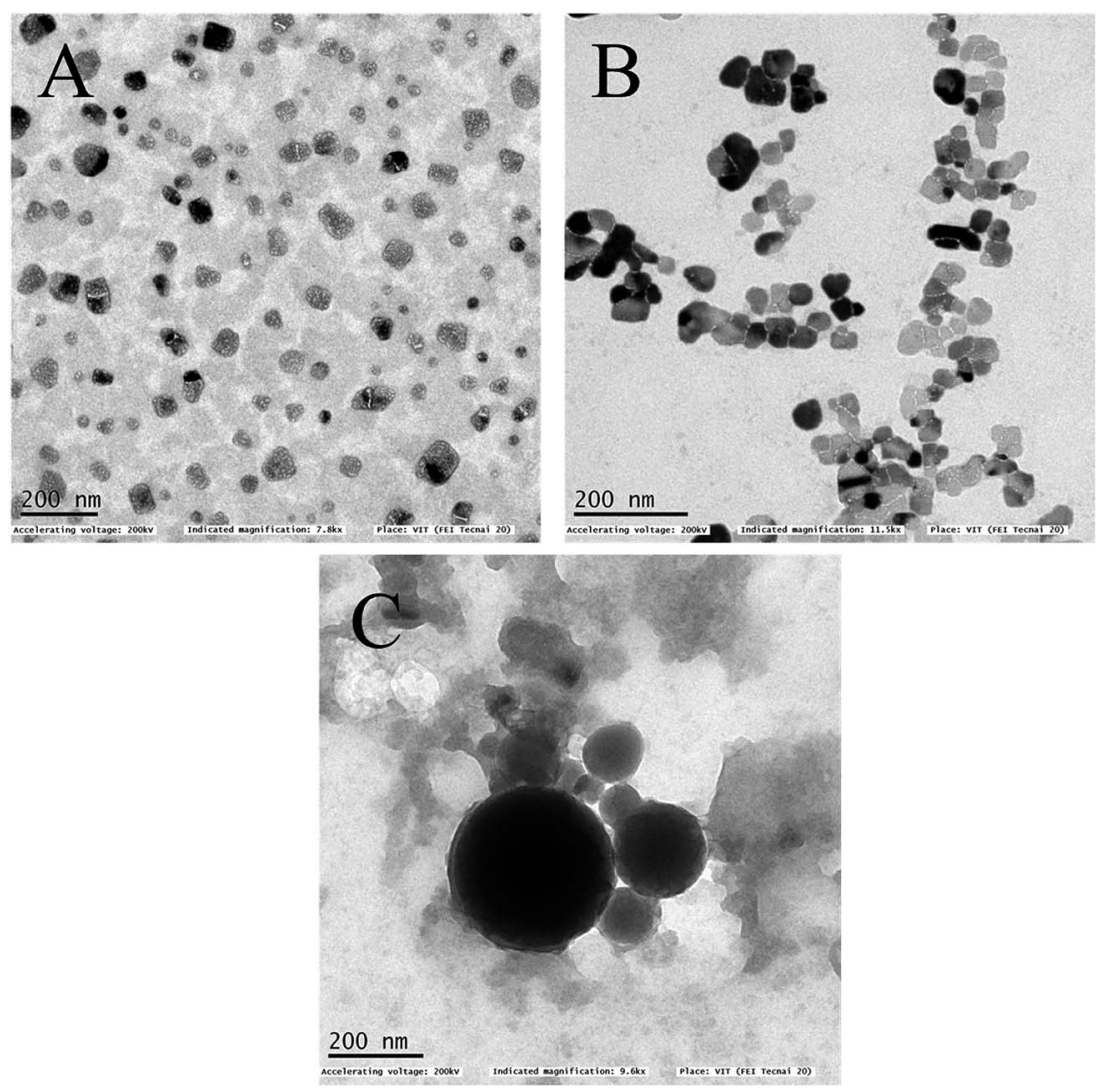

Fig. 3 TEM image of (A) as extracted seaweed polysaccharide (B) orange oil nanoemulsion encapsulated with seaweed polysaccharide (C) NLC encapsulated with seaweed polysaccharide. 

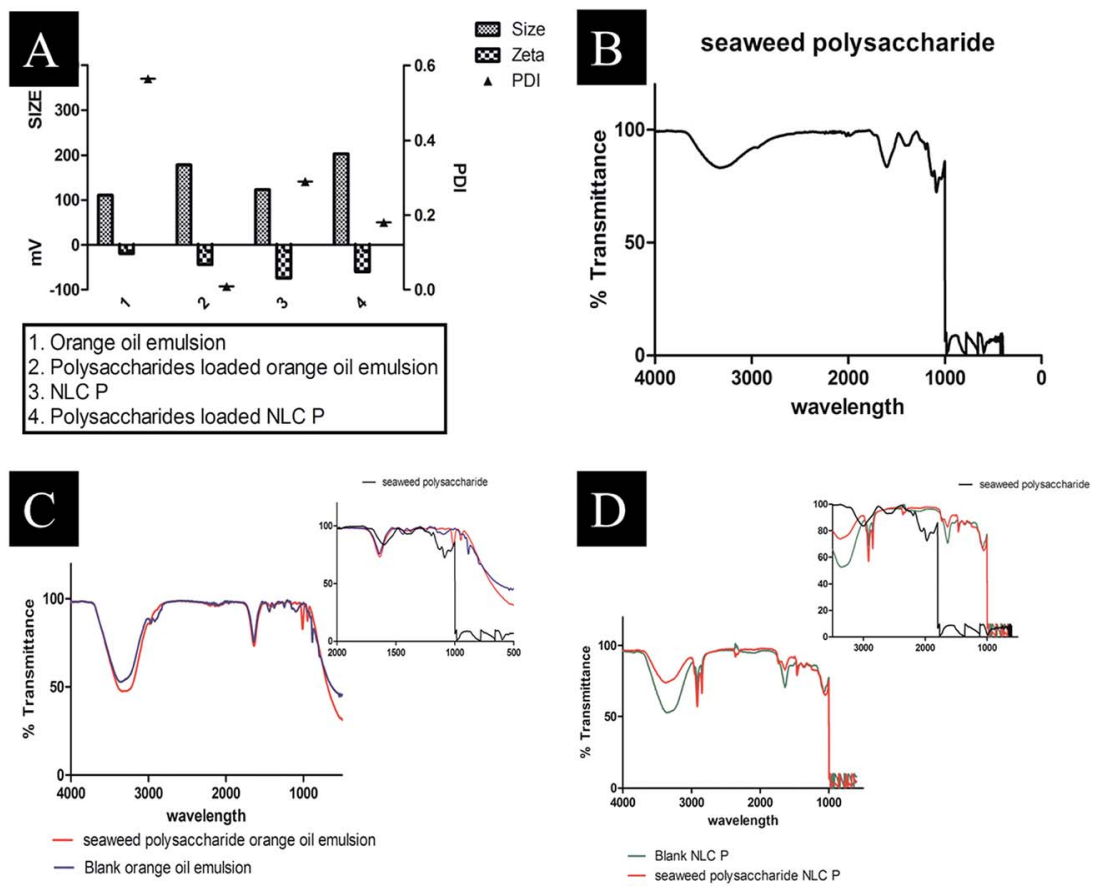

Fig. 4 (A) DLS size distribution of prepared formulations (B) FTIR spectra of seaweed polysaccharide (C) FTIR spectra of blank orange oil nanoemulsion and seaweed polysaccharide loaded orange oil nanoemulsion (D) FTIR spectra of blank NLC and seaweed polysaccharide loaded NLC.

scattering (DLS). The obtained values are tabulated in Table 4 and Fig. 4A. The particle size distribution of crude seaweed polysaccharide loaded orange oil nanoemulsion was $178 \mathrm{~nm}$ with PI 0.009 and zeta potential $-43.9 \mathrm{mV}$. NLC loaded with crude polysaccharide ranged with size $203 \mathrm{~nm}, 0.18$ PI and $-60 \mathrm{mV}$ zeta potential.

\subsection{SEM and TEM}

The surface morphologies of the formulated samples were examined using scanning electron microscopy (SEM) and transmission electron microscopy (TEM). Crude seaweed polysaccharide (Fig. 2A and 3A) showed irregular shape which is highly undesirable for biomedical applications. But the nanocarrier images relatively smoother surface morphology.

Table 5 List of infrared (IR) vibrational modes characteristic to polysaccharides

\begin{tabular}{lll}
\hline & Seaweed polysaccharide & \\
\cline { 2 - 3 } Frequency & Bond & Functional groups \\
\hline 3327.21 & O-H stretch & Alcohol \\
2939.52 & O-H stretch & Carboxylic acids \\
1600.92 & C-C stretch & Carbonyl group \\
1381.03 & SO & Sulphate groups \\
1126.43 & C-O stretch & Carboxylic acids \\
1087.85 & RO-SO & Sulphate groups \\
1037.70 & RO-SO & Sulphate groups \\
650.01 & C-Cl stretch & Alkyl halides \\
597.93 & C-Br stretch & Alkyl halides
\end{tabular}

Nanoemulsion (Fig. 2B and 3B) shape was not fully spherical but its clear shape and size adds to its advantage. NLC (Fig. 2C and $3 \mathrm{C}$ ) was fully spherical with very small size proving to be the best suitable nanocarrier.

\subsection{Fourier transform infra-red spectroscopy (FTIR)}

Functionality of seaweed polysaccharide loaded and unloaded in nanoemulsion and NLCs were shown in (Fig. 4B-D). It is observed that the presence of functional groups in both the cases is the same, showing that nanoemulsion and nanostructured carrier processing did not change the chemical functionality of the polysaccharides. The characteristic peaks for seaweed polysaccharide are $1381 \mathrm{~cm}^{-1}, 1126 \mathrm{~cm}^{-1}$, $1087 \mathrm{~cm}^{-1}, 1037 \mathrm{~cm}^{-1}, 650 \mathrm{~cm}^{-1}, 597 \mathrm{~cm}^{-1}$ and $482 \mathrm{~cm}^{-1}$ (Table 5) while the characteristic peaks for orange oil is similar to limonene $1641 \mathrm{~cm}^{-1}, 1438 \mathrm{~cm}^{-1}$ and $885 \mathrm{~cm}^{-1}$. The polysaccharides peaks are not found in the loaded one and thus it indicates that the polysaccharide has been encapsulated into emulsion (Table 6). The characteristic peaks for NLC are $1639 \mathrm{~cm}^{-1}, 1465 \mathrm{~cm}^{-1}, 1350 \mathrm{~cm}^{-1}, 1056 \mathrm{~cm}^{-1}$, and $885 \mathrm{~cm}^{-1}$. The corresponding seaweed polysaccharide peaks are absent in the loaded NLC which also indicates that the polysaccharide has been encapsulated into NLC (Table 7).

\subsection{Entrapment efficiency and release study}

Entrapment efficiency of the formulated nanocarriers was calculated using eqn (1) and found that seaweed polysaccharide loaded orange oil nanoemulsion was $67.29 \pm 2 \%$ (Fig. 5A) and in seaweed polysaccharide loaded NLC was $78.7 \pm 2 \%$ (Fig. 5B). 
Table 6 Characteristic peaks of blank and seaweed polysaccharide loaded orange oil nanoemulsion

\begin{tabular}{|c|c|c|c|c|}
\hline \multirow[b]{2}{*}{ Frequency } & \multicolumn{2}{|c|}{ Blank orange oil } & \multicolumn{2}{|c|}{$\begin{array}{l}\text { Seaweed polysaccharide } \\
\text { loaded orange oil nanoemulsion }\end{array}$} \\
\hline & Bond & Functional groups & Bond & Functional groups \\
\hline 3360 & $\mathrm{O}-\mathrm{H}$ stretch & Alcohol & O-H stretch & Alcohol \\
\hline 2966.52 & & & O-H stretch & Carboxylic acids \\
\hline 2922.16 & $\mathrm{O}-\mathrm{H}$ stretch & Carboxylic acids & & \\
\hline 1641.42 & $-\mathrm{C}=\mathrm{C}-$ stretch & Alkenes & & \\
\hline 1637.56 & & & $\mathrm{~N}-\mathrm{H}$ bend & Primary amine \\
\hline 1438.90 & C-C stretch & Aromatics & & \\
\hline 1249.94 & $\mathrm{C}-\mathrm{N}$ stretch & Aromatic amines & & \\
\hline 1097.50 & C-N stretch & Aliphatic amines & & \\
\hline 1010.70 & & & $\mathrm{C}-\mathrm{O}$ stretch & $\begin{array}{l}\text { Alcohols, carboxylic } \\
\text { acids, esters, ethers }\end{array}$ \\
\hline 948.98 & & & O-H stretch & Carboxylic acids \\
\hline 885.33 & $\mathrm{C}-\mathrm{H}$ stretch & Aromatics & & \\
\hline 790.81 & C-Cl stretch & Alkyl halides & & \\
\hline 538.14 & C-Br stretch & Alkyl halides & & \\
\hline
\end{tabular}

Thus keeping $80 \%$ as control for release study seaweed polysaccharide from orange oil nanoemulsion and NLC formulations were analyzed in vitro in enzyme free simulated intestinal medium. Over a period of $12 \mathrm{~h} \sim 80 \%$ of seaweed polysaccharide was released from orange oil nanoemulsion and $\sim 95 \%$ was released from NLC. Slow and sustained release of seaweed polysaccharide was noted (Fig. 5C and D). From the results high entrapment and more amount of seaweed polysaccharide release was seen in NLC when compared to nanoemulsion formulation.

\subsection{Cytotoxic activity}

The cytotoxicity activity of seaweed polysaccharide encapsulated with nanoemulsion and NLC was analyzed with MTT assay against normal mouse fibroblast 3T3 (Fig. 6) and colon cancer cell lines HCT 116 (Fig. 7). The cytotoxic activity was performed for seaweed polysaccharide, orange oil, biosurfactants, nanoemulsion and NLC. From the results we infer that the seaweed polysaccharide, emulsion and NLC exhibited no inhibitory effect on normal mouse fibroblast cell lines $(99.9 \pm 0.05 \%$ viability), whereas seaweed polysaccharide alone provides cytotoxicity activity against HCT 116 cells but the activity was more efficient when loaded into nanoemulsion and NLC nanocarriers. The percentage of cell viability in seaweed polysaccharide loaded NLC is $23 \pm 0.7 \%$ at $1000 \mu \mathrm{g} \mathrm{ml}{ }^{-1}$. $80 \%$ of cells was killed at this concentration. At the same concentration seaweed polysaccharide loaded nanoemulsion kills $70 \pm 0.4 \%$ of cells whereas the seaweed polysaccharide alone kills $55 \pm$ $0.6 \%$. Thus seaweed polysaccharide encapsulated with NLC has better efficiency when compared to nanoemulsion and seaweed polysaccharide alone (Fig. 8).

Table 7 Characteristic peaks of blank and seaweed polysaccharide loaded NLC

\begin{tabular}{|c|c|c|c|c|}
\hline \multirow[b]{2}{*}{ Frequency } & \multicolumn{2}{|l|}{ Blank NLC } & \multicolumn{2}{|c|}{ Seaweed polysaccharide loaded NLC } \\
\hline & Bond & Functional groups & Bond & Functional groups \\
\hline 3375.43 & & & $\mathrm{O}-\mathrm{H}$ stretch & Alcohol \\
\hline 3363.86 & $\mathrm{O}-\mathrm{H}$ stretch & Alcohol & & \\
\hline 2916.37 & $\mathrm{O}-\mathrm{H}$ stretch & Carboxylic acids & $\mathrm{O}-\mathrm{H}$ stretch & Carboxylic acids \\
\hline 2850.79 & C-H stretch & Alkanes & C-H stretch & Alkanes \\
\hline 2360.87 & $\mathrm{C} \equiv \mathrm{N}$ stretch & Nitriles & $\mathrm{C} \equiv \mathrm{N}$ stretch & Nitriles \\
\hline 1732.08 & & & $\mathrm{C}=\mathrm{O}$ stretch & Carbonyls \\
\hline 1639.49 & $-\mathrm{C}=\mathrm{C}-$ stretch & Alkenes & $-\mathrm{C}=\mathrm{C}-$ stretch & Alkenes \\
\hline 1512.19 & & & $\mathrm{~N}-\mathrm{O}$ asymmetric stretch & Nitro compounds \\
\hline 1465.90 & C-C stretch (in-ring) & Aromatics & C-C stretch (in-ring) & Aromatics \\
\hline 1369.46 & & & C-H rock & Alkanes \\
\hline 1350.17 & $\mathrm{C}-\mathrm{H}$ rock & Alkanes & & \\
\hline 1219.01 & & & $-\mathrm{CH}_{2} \mathrm{X}$ & Alkyl halides \\
\hline 1056.99 & C-O stretch & $\begin{array}{l}\text { Alcohols, carboxylic } \\
\text { acids, esters, ethers }\end{array}$ & C-O stretch & $\begin{array}{l}\text { Alcohols, carboxylic } \\
\text { acids, esters, ethers }\end{array}$ \\
\hline 721.38 & & & $\mathrm{C}-\mathrm{H}$ rock & Alkanes \\
\hline
\end{tabular}


A

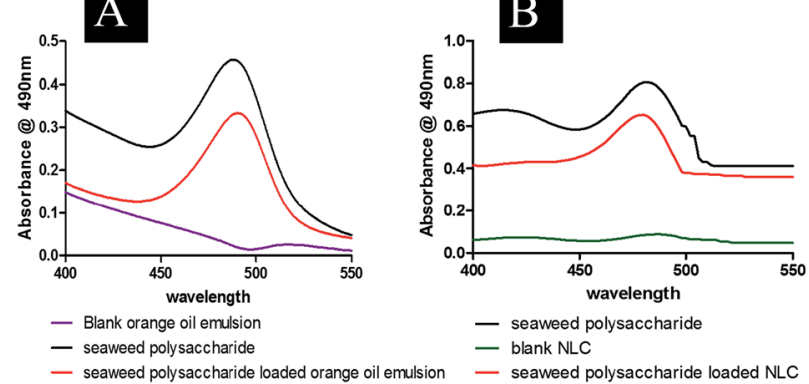

Fig. 5 Encapsulation efficiency of (A) seaweed polysaccharide in orange oil nanoemulsion (B) seaweed polysaccharide in NLC.

\section{Discussion}

\subsection{Extraction and yield of water soluble polysaccharides}

In this study, extraction of crude seaweed polysaccharides was done according to the procedure of ref. 21 and 22 with slight modification. The seaweed extract was boiled in distilled water at $100{ }^{\circ} \mathrm{C}$ by adjusting the $\mathrm{pH}$ to 2 with hydrochloric acid $(\mathrm{HCl})$. The sample was allowed to boil for $3 \mathrm{~h}$ and the supernatant was collected and precipitated with $100 \%$ ethanol to obtain high yield of crude polysaccharides. The result obtained was similar to the studies reported by ref. 31 because of the parameters used in the hot water extraction method. The yield of crude polysaccharide from brown seaweed Sargassum longifolium in this study was higher $(10.38 \%)$ than in previous reports for $S$. latifolium $(4.75 \%),{ }^{29}$ S. fulvellum (8.9\%) and $S$. thunbergii $(9.6 \%){ }^{32}$ The difference in the yield compared to other reports might be because of the higher acid concentration $\left(2 \mathrm{~mol} \mathrm{l}^{-1} \mathrm{HCl}\right)$ used in the present study. The other reason for low yield is due to the different solubility of the polysaccharides in the solvents. ${ }^{33}$

\subsection{Importance of nanocarriers}

Marine polysaccharides have different biomedical applications and have drawn its attention in the present research. Seaweed derived polysaccharides has extended its potential activities against cancer and virus. Polysaccharides, the major bioactive compound of seaweeds have a wide role in nutraceutical and medical applications. ${ }^{13}$ Though seaweed polysaccharides are being used in a list of applications, the efficiency is still low

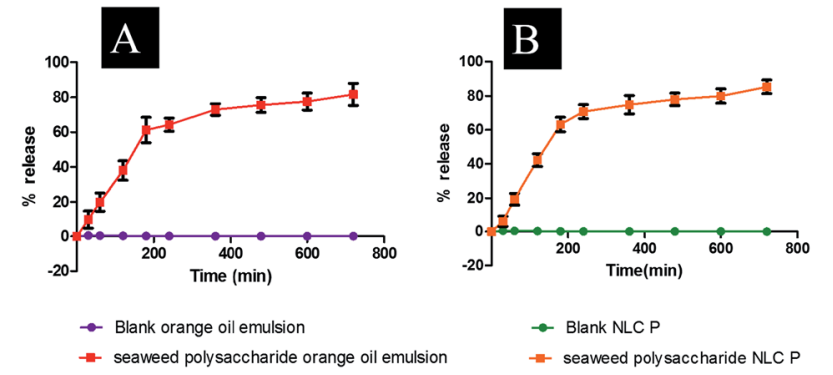

Fig. 6 Release study of seaweed polysaccharide from (A) orange oil nanoemulsion (B) NLC.

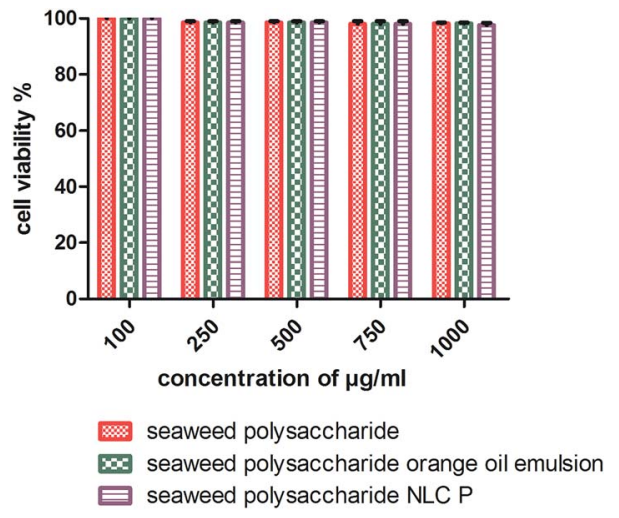

Fig. 7 Cell viability of unloaded and loaded seaweed polysaccharide in orange oil nanoemulsion and NLC in 3T3 mouse normal fibroblast cell lines.

because of its large size and irregular shape. Till date there are so many reports stating that polysaccharides from seaweeds have a potent role in treating various cancers. The conventional mean of drug administration is oral route for patients requiring long term treatment. Hence nanoparticle approach for natural products has been explored. Natural products encapsulated in inert systemic nanocarriers tend to be more active and efficient than their original nature because of their small size, easy penetration into the cells and cell organelles, large surface area, enhanced bioavailability and long term stability. ${ }^{34}$ Advantage of using nanoemulsion as a nanocarrier is that it protects the encapsulated compound from hydrolysis and oxidation. It is also considered to be effective and safe with increased bioavailability. ${ }^{35}$ NLC is a nanocarrier with the same advantages of nanoemulsion but because of its disrupted matrices, the major advantage of NLC leads to higher drug entrapment and sustained release which ultimately enhances drug absorption when compared with other lipid based formulations having uniform matrices of lipids. ${ }^{36}$ In our study the efficiency of both nanoemulsion and NLC nanocarriers encapsulated with seaweed derived polysaccharides has been studied and compared.

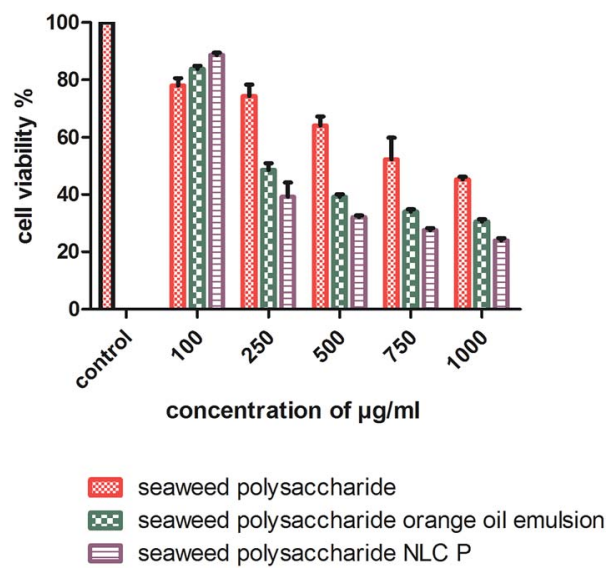

Fig. 8 Cell viability and proliferation of unloaded and loaded seaweed polysaccharide in orange oil nanoemulsion and NLC in HCT 116 colon cancer cell lines. 


\subsection{Size distribution and zeta potential}

In Table 4 the particle size distribution of the nanocarrier formulation is tabulated. In order to improve the bioavailability of the seaweed polysaccharide, nanoemulsion and NLC nanocarrier encapsulated with seaweed polysaccharide were formulated using ultra sonication and hot solvent diffusion method respectively. The main role of nanocarriers is their size as it determines the bio accessibility of the entrapped compounds by affecting the gastrointestinal tract residence time, dissolution rate and action of digestive enzymes. ${ }^{37}$ From the literature it was noted that generally particles with $<200 \mathrm{~nm}$ are preferred for oral delivery. Particle size analysis confirms that all the formulations were within the required range. The particle size and PI of orange oil nanoemulsion was $111 \mathrm{~nm}$ and 0.5 whereas for NLC $153 \mathrm{~nm}$ and 0.18 respectively. Addition of seaweed polysaccharide to the nanocarriers increased the particle size but PI was decreased. This increase in particle size indicates the incorporation of seaweed polysaccharide into the formulated matrix and decrease in PI reveals the homogenous distribution of the particle in the media. The surface charge density (zeta potential) represents the in vitro stability of the formulated nano carriers. The preferable range for determining good stability is more than $\pm 30 \mathrm{mV}$. The surface charge densities of the nanoemulsion and NLC (blank and seaweed polysaccharide loaded) indicated excellent stability as it ranged from $-30 \mathrm{mV}$ to $-60 \mathrm{mV}$. Similar study was reported ${ }^{38}$ where NLC prepared by solvent diffusion method using stearic acid and oleic acid showed size ranging from $150 \mathrm{~nm}$ to $380 \mathrm{~nm}$ in blank and in drug loaded NLC $160 \mathrm{~nm}$ to $430 \mathrm{~nm}$. The PI of this fabricated NLC ranges from 0.005 to 0.4 and zeta potential from -40 to $-50 \mathrm{mV}$.

\subsection{Entrapment and release study}

Seaweed polysaccharide is considered as a nutraceutical because it is used both as a food and medicine. But to show its potent therapeutic benefit nanosize of this seaweed polysaccharide is found to be successful by encapsulating it into nano carriers. From the literature it was stated that ${ }^{38}$ the drug entrapment efficiency in NLC prepared by solvent diffusion method ranged from 45 to $70 \%$. Ref. 39, 40 and 41 resulted that the embodiment of liquid lipids to solid lipids could lead to enormous crystal order disturbance, and the resulting matrix of lipid particles indicates deformity in the crystal lattice and leaves adequate space to attune drug molecules, thus, leading to enhanced drug entrapment efficiency. Reports stated that Antrodia camphorata polysaccharides encapsulated in silica chitosan nanoparticles showed encapsulation efficiency $66 \%$ and with silica nanoparticles alone was $63.5 \% .^{42}$ Thus high entrapment efficiency is observed due to the lipophilic nature of our fabricated nanocarriers with $67.29 \pm 2 \%$ in nanoemulsion and $78.7 \pm 2 \%$ in NLC. High entrapment of seaweed polysaccharide was seen in NLC when compared to nanoemulsion. This may be because of the irregular matrix of the NLC which incorporates more amounts of compounds.

The release of seaweed polysaccharide from nanoemulsion and NLC was tested in enzyme free SIM (simulated intestinal medium). Gastrointestinal tract is an unfavorable environment for seaweed polysaccharide as it may degrade before reaching the intestine. Thus, seaweed polysaccharide is protected by encapsulating into nanocarriers so it passes safely until it reaches the intestine. In the intestine the formulated nanocarriers form into a micelle like structure as it is composed of bile salts, phospholipids and lipid degradation products like monoglycerides, fatty acids etc. ${ }^{\mathbf{4 3}}$ The seaweed polysaccharide release behavior from nanocarriers (nanoemulsion and NLC) did not exhibit a biphasic pattern with burst release initially followed by sustained release. The release in our study was controlled release from the beginning and high amount of release was seen from NLC compared to nanoemulsion. This may be because of the lecithin which helps in hydrolysis of the formulated NLC by digestive enzymes. ${ }^{38}$

\subsection{Cytotoxic activity against normal mouse fibroblast cells and colon cancer cell lines}

Polysaccharides from brown seaweed cannot be hydrolyzed by digestive enzymes in the human small intestine and therefore it proves to be efficient compound to prevent colon carcinogenesis. ${ }^{31}$ It becomes necessary to study the toxicity of anti-cancer compounds in normal cells as the compound should not compromise normal cells. The error bars for normal cell line 3T3 mouse fibroblast cells is very low because the values were very close to the control (more than $95 \%$ of cells were viable). The compounds (seaweed polysaccharide, orange oil nanoemulsion and NLC) were non-toxic to 3T3 mouse fibroblast normal cell line. Similar results have been found in human normal dermal fibroblasts cell line in the presence of betulinic acid obtained from various traditional plants. ${ }^{\mathbf{4 4}}$ Thus, these compounds seem to be selective for tumor cells which matches with the very minimal toxicity against normal cells observed during our study. Previous studies have reported that seaweed polysaccharides exhibit anti-cancer effects, metastasis and angiogenesis in various cancer cells. ${ }^{\mathbf{1 1 4 5}}$ Studies have reported that fucoidan induced apoptosis in HCT-15 human colon cancer cells at a concentration of $100 \mu \mathrm{g} \mathrm{ml}{ }^{-1}$. In this study crude seaweed polysaccharide kills $55 \%$ of cancer cells at 1000 $\mu \mathrm{g} \mathrm{ml}{ }^{-1}$. But nanocarriers loaded with crude polysaccharides killed $70-80 \%$ of cancer cells proving to be more efficient. The cell viability test was also done for individual components of nanocarriers like orange oil, biosurfactants stearic acid. At low concentrations $\left(10-20 \mu \mathrm{g} \mathrm{ml}^{-1}\right)$ these components exerts anticancer activity by killing cancer cells. But at higher concentration $\left(>50 \mu \mathrm{g} \mathrm{ml}^{-1}\right)$ it helps in proliferating the cancer cells thus inhibiting apoptosis. But the formulated nanocarriers with seaweed polysaccharide possessed efficient activity against HCT 116 cells even at higher concentrations upto $1 \mathrm{mg} \mathrm{ml}^{-1}$. Studies have reported that NLCs loaded with bioactive compounds exhibited enhanced cell growth inhibition in in vitro cell viability assay when compared to unloaded bioactive compounds. ${ }^{29}$ The mechanism involved in the selective and reduced toxicity of compounds to normal cells as compared to tumor cells is that cancer cells which are capable of rapid proliferation are more effectively killed by these compounds 
whereas the normal cell populations which have lower proportion of cells remain alive because of their slow entry into the $\mathrm{S}$ phase of cell cycle. This selective toxicity is because the compounds reduce the saturation density of untransformed cells (normal cells) thus arresting them in the G1 phase of the cell cycle. ${ }^{46}$

\section{Conclusions}

In summary, encapsulation of seaweed polysaccharides in orange oil nanoemulsion and NLC exhibited successfully and showed significantly increased cytotoxicity against colon cancer cell lines HCT 116. From the results we infer that the seaweed polysaccharide encapsulated in NLC formulated by hot solvent diffusion method has high entrapment efficiency with reliable particle size and zeta potential when compared to orange oil nanoemulsion and the release profile of polysaccharide from NLC was high with sustained pattern compared to nanoemulsion. Though both the nanocarriers encapsulated with seaweed polysaccharide exhibited good anticancer activity, the NLC takes a better place when compared with nanoemulsion. Therefore, encapsulating seaweed polysaccharides in nanocarriers illustrates a promising approach to provide cytotoxic activity with improved efficacy and safety.

\section{Conflicts of interest}

There are no conflicts of interest.

\section{Acknowledgements}

The authors are grateful for the support provided by VIT University and Madurai Kamaraj University to carry out the research work. Saghya Infant Shofia acknowledges the Maulana Azad National Fellowship (UGC-MANF) for the financial support (F117.1/201516/MANF201517PON48888/(SAIII/Website)).

\section{References}

1 A. J. Smit, J. Appl. Phycol., 2004, 16, 245-262.

2 K. H. Cardozo, T. Guaratini, M. P. Barros, V. R. Falcão, A. P. Tonon, N. P. Lopes, S. Campos, M. A. Torres, A. O. Souza and P. Colepicolo, Comp. Biochem. Physiol., Part C: Toxicol. Pharmacol., 2007, 146, 60-78.

3 K.-X. Yu, I. Jantan, R. Ahmad and C.-L. Wong, Parasitol. Res., 2014, 113, 3121-3141.

4 Singh A., Bhat T. K. and Sharma O. P., Clinical biochemistry of hepatotoxicity, J. Clin. Toxicol., 2011, 4(001), 1-19.

5 H. Stegenga, Bot. Mar., 2011, 54, 109.

6 M. T. Ale and A. S. Meyer, RSC Adv., 2013, 3, 8131-8141.

7 L. G. Ferreira, M. D. Noseda, A. G. Gonçalves, D. R. Ducatti, M. T. Fujii and M. E. Duarte, Carbohydr. Res., 2012, 347, 8394.

8 J. Venkatesan, S. Anil, S.-K. Kim and M. S. Shim, Polymers, 2016, 8, 30.

9 W. Black, J. Mar. Biol. Assoc. U. K., 1954, 33, 49-60.
10 M. T. Ale, H. Maruyama, H. Tamauchi, J. D. Mikkelsen and A. S. Meyer, Int. J. Biol. Macromol., 2011, 49, 331-336.

11 E. J. Kim, S. Y. Park, J.-Y. Lee and J. H. Y. Park, BMC Gastroenterol., 2010, 10, 96.

12 Y. Yamasaki-Miyamoto, M. Yamasaki, H. Tachibana and K. Yamada, J. Agric. Food Chem., 2009, 57, 8677-8682.

13 N. Ruocco, S. Costantini, S. Guariniello and M. Costantini, Molecules, 2016, 21, 551.

14 M. S. Kim, J. Y. Kim, W. H. Choi and S. S. Lee, Nutr. Res. Pract., 2008, 2, 62-67.

15 H. Ye, K. Wang, C. Zhou, J. Liu and X. Zeng, Food Chem., 2008, 111, 428-432.

16 A. Jiménez-Escrig and F. Sánchez-Muniz, Nutr. Res., 2000, 20, 585-598.

17 L. Montenegro, F. Lai, A. Offerta, M. G. Sarpietro, L. Micicchè, A. M. Maccioni, D. Valenti and A. M. Fadda, J. Drug Delivery Sci. Technol., 2016, 32, 100-112.

18 K. Bouchemal, S. Briançon, E. Perrier and H. Fessi, Int. J. Pharm., 2004, 280, 241-251.

19 F. Donsì and G. Ferrari, J. Biotechnol., 2016, 233, 106-120.

20 Y. Chang and D. J. McClements, J. Agric. Food Chem., 2014, 62, 2306-2312.

21 T. Ozawa, J. Yamamoto, T. Yamagishi, N. Yamazaki and M. Nishizawa, J. Nat. Med., 2006, 60, 236-239.

22 A. Kantachumpoo and A. Chirapart, Kasetsart J.: Nat. Sci., 2010, 44, 220-233.

23 K. F. Akhter, M. A. Mumin, E. K. Lui and P. A. Charpentier, ACS Biomater. Sci. Eng., 2015, 2, 96-103.

24 Z. Du, C. Wang, X. Tai, G. Wang and X. Liu, ACS Sustainable Chem. Eng., 2016, 4, 983-991.

25 C. L. Dora, J.-L. Putaux, I. Pignot-Paintrand, F. Dubreuil, V. Soldi, R. Borsali and E. Lemos-Senna, J. Braz. Chem. Soc., 2012, 23, 1972-1981.

26 Y. Liu, F. Wei, Y. Wang and G. Zhu, Colloids Surf., A, 2011, 389, 90-96.

27 P. Izquierdo, J. Feng, J. Esquena, T. F. Tadros, J. C. Dederen, M. J. Garcia, N. Azemar and C. Solans, J. Colloid Interface Sci., 2005, 285, 388-394.

28 A. K. Sachan, A. Gupta and M. Arora, J. Drug Delivery Ther., 2016, 6, 4-13.

29 N. Aditya, M. Shim, I. Lee, Y. Lee, M.-H. Im and S. Ko, J. Agric. Food Chem., 2013, 61, 1878-1883.

30 X.-Y. Yang, Y.-X. Li, M. Li, L. Zhang, L.-X. Feng and N. Zhang, Cancer Lett., 2013, 334, 338-345.

31 M. Mohsen, S. F. Mohamed, F. Ali and O. H. El-Sayed, J. Appl. Sci. Res., 2007, 3, 1178-1185.

32 J. Kang, M. Khan, N. Park, J. Cho, M. Lee, H. Fujii and Y. Hong, J. Ethnopharmacol., 2008, 116, 187-190.

33 A. Usov, G. Smirnova, Z. Kamenarska, K. Stefanov and S. Popov, Russ. J. Bioorg. Chem., 2004, 30, 161-167.

34 R. Kimura, T. Rokkaku, S. Takeda, M. Senba and N. Mori, Mar. Drugs, 2013, 11, 4267-4278.

35 M. Jaiswal, R. Dudhe and P. Sharma, 3 Biotech, 2015, 5, 123127.

36 S. Khan, S. Baboota, J. Ali, S. Khan, R. S. Narang and J. K. Narang, Int. J. Pharm. Invest., 2015, 5, 182. 
37 P. Severino, T. Andreani, A. S. Macedo, J. F. Fangueiro, M. H. A. Santana, A. M. Silva and E. B. Souto, J. Drug Delivery, 2012, 2012, 750891.

38 F.-Q. Hu, S.-P. Jiang, Y.-Z. Du, H. Yuan, Y.-Q. Ye and S. Zeng, Colloids Surf., B, 2005, 45, 167-173.

39 V. Jenning, A. F. Thünemann and S. H. Gohla, Int. J. Pharm., 2000, 199, 167-177.

40 V. Jenning and S. H. Gohla, J. Microencapsulation, 2001, 18, 149-158.

41 E. Souto, S. Wissing, C. Barbosa and R. Müller, Int. J. Pharm., 2004, 278, 71-77.
42 Z.-L. Kong, J.-S. Chang and K. L. B. Chang, J. Nanopart. Res., 2013, 15, 1945.

43 K. R. Walsh, Y. C. Zhang, Y. Vodovotz, S. J. Schwartz and M. L. Failla, J. Agric. Food Chem., 2003, 51, 4603-4609.

44 V. Zuco, R. Supino, S. C. Righetti, L. Cleris, E. Marchesi, C. Gambacorti-Passerini and F. Formelli, Cancer Lett., 2002, 175, 17-25.

45 J.-H. Hyun, S.-C. Kim, J.-I. Kang, M.-K. Kim, H.-J. Boo, J.-M. Kwon, Y.-S. Koh, J.-W. Hyun, D.-B. Park and E.-S. Yoo, Biol. Pharm. Bull., 2009, 32, 1760-1764.

46 E. Rozengurt and C. C. Po, Nature, 1976, 261, 701. 